\title{
Madrid en huelga, Madrid sin agua: el desabastecimiento de agua en Madrid a consecuencia de la huelga de la construcción de 1936 a través de las fuentes hemerográficas
}

\author{
Madrid on strike, Madrid without water: the shortage of water in Madrid as a \\ result of the 1936 construction strike through the hemographic sources.
}

\author{
Ruy Rodero Torrealba \\ Universidad Complutense de Madrid \\ rrodero@ucm.es
}

Fecha de recepción: 14 de febrero de 2019

Fecha de aceptación: 2 de agosto de 2019

\begin{abstract}
Resumen
En los meses de junio y julio/agosto de 1936 se desató en la ciudad de Madrid una colosal huelga en el sector de la construcción que dejó sin agua a una parte importante de los vecinos de la capital. Calles y barrios de la ciudad sufrieron la falta de tan preciado líquido en los meses que el calor más aprieta, viéndose así perturbada la vida de los ciudadanos. La prensa se hizo eco de la situación que vivían los madrileños y no dudó en atacar a las organizaciones sindicales, el Ayuntamiento y al Estado. La inicial intransigencia de los huelguistas se fue suavizando a medida que se hacía más insoportable convivir con el desabastecimiento de agua.
\end{abstract}

Palabras clave: Madrid, huelga de la construcción, desabastecimiento de agua, primavera de 1936.

\begin{abstract}
In the months of June and July/August 1936 a colossal strike in the construction sector was unleashed in the city of Madrid, which left a significant part of the capital's residents without water. Streets and neighborhoods of the city suffered the lack of such a precious liquid in the months that the heat suffocates the most, thus it disturbed the life of the citizens. The press echoed the situation experienced by the people of Madrid and did not hesitate to attack the trade unions, the City Council and the State. The initial intransigence of the strikers was softened as it became more unbearable to live with said shortage of water.
\end{abstract}

Key words: Madrid, construction strike, water shortage, spring 1936. 
Madrid en huelga, Madrid sin agua: el desabastecimiento de agua en Madrid a consecuencia de la huelga de la construcción de 1936 a través de las fuentes hemerográficas

\section{1-Introducción}

Madrid, capital de la República y ciudad con más de un millón de habitantes a la altura de $1936^{1}$, vivió en los meses de junio-agosto de ese año una colosal huelga en el sector de la construcción que afectó a más de 80000 obreros $^{2}$. Movimiento huelguístico de tal magnitud, que pronto se extendió a otros sectores ${ }^{3}$ y acabó afectando la vida cotidiana de una buena parte de los madrileños a raíz del desabastecimiento de agua en numerosos puntos de la ciudad ${ }^{4}$. El presente escrito busca abordar la cuestión del desabastecimiento de agua a consecuencia de la huelga de la construcción e intentar responder a ciertas cuestiones (¿a qué zonas de Madrid afectó la falta de agua?, ¿durante cuánto tiempo?, ¿cuál fue la posición adoptada por las autoridades municipales?, ¿y por las organizaciones sindicales?), dado que es algo que se ha dejado de lado en la bibliografía existente sobre este conflicto urbano ${ }^{5}$. Para el acercamiento al desabastecimiento de agua se recurrirá a la prensa del momento, fuente fundamental a la hora de estudiar la historia contemporánea pues, siguiendo a Tuñón de Lara, «sin contar con la historia de la prensa no es posible hoy en día "hacer" historia contemporánea, es decir, investigar en ella, comprenderla y explicarla» ${ }^{6}$.

El interés por el estudio del desabastecimiento de agua en la ciudad de Madrid viene motivado por diversas cuestiones. En primer lugar, por el hecho de ser algo que no se ha reflejado en la bibliografía existente sobre la conflictividad sociolaboral en Madrid durante la primavera de 1936; en segundo lugar, porque la falta de agua se produce en la capital de la República, con las repercusiones que eso tiene, y a las puer-

$1 \quad$ Madrid tenía una población de hecho de 1041767 habitantes en 1930 y de 1322835 en 1940. Instituto de Estadística, Estadísticas Históricas. Censos y padrones. Población de la Comunidad de Madrid por municipios. Consultado el día 5 de febrero de 2019, http://www.madrid.org/iestadis/fijas/estructu/general/ otros/iserieshistoricas_pob.htm

2 Francisco Sánchez Pérez, "Clase obrera y conflictividad social en el Madrid del Frente Popular (febrero-julio de 1936)", Cuadernos de Historia Contemporánea 13 (1991): 68; Santos Juliá Díaz, "¿Feudo de la UGT o capital confederal? La última huelga de la construcción en el Madrid de la República", Historia Contemporánea 6 (1991): 216; Julián Vadillo Muñoz, "Entre el pacto y la revolución. El movimiento libertario en la primavera de 1936", Nuestra Historia: revista de Historia de la FIM 1 (2016): 63. Esta cifra, que asumimos como correcta, aparece en la prensa de la época ("Ochenta mil trabajadores llevan ya diecisiete días de huelga. Urge que se le ponga remedio", El Socialista, 18 de junio de 1936, 5 y "La situación social derivada de las huelgas", La Vanguardia, 26 de junio de 1936, 28), aunque en ocasiones se eleva hasta casi 100000 (Indalecio Prieto, "Enseñanzas de una huelga", El Liberal (Bilbao), 23 de junio de 1936, 1) o 120000 ("La huelga de la construcción ha entrado en la séptima semana", La Vanguardia, 15 de julio de 1936, 23), seguramente siendo exageraciones al ser artículos bastante críticos con el proceder de los huelguistas.

3 Sánchez Pérez, "Clase obrera y conflictividad social", 68.

4 Francisco Sánchez Pérez, "La huelga de la construcción en Madrid (junio-julio, 1936)", Historia 16 154 (1989): 24.

5 «Diversas barriadas quedaron sin agua» (Sánchez Pérez, "La huelga de la construcción en Madrid", 24) es todo lo que aparece en la bibliografía consultada.

6 Manuel Tuñón de Lara, "Prensa obrera e historia contemporánea", en: Prensa Obrera en Madrid 18551936, ed. Santiago Castillo y Luis E. Otero Carvajal (Madrid: Comunidad de Madrid y Alfoz, 1987), 29. 
tas del verano o ya entrado en él, cuando el calor más aprieta y más necesario se hace disponer de tan preciado líquido, siempre con el temor a la propagación de alguna epidemia, tal y como se advertía en la prensa de la época ${ }^{7}$; y por último, por la fatiga de tener que hacer largas colas para conseguir el agua de los tanques municipales, las bocas de riego o las fuentes públicas, lo que se veía incrementado por el hecho de que muchos ascensores de la ciudad no funcionaban a causa de la huelga de los ascensoristas $^{8}$, obligando a los vecinos a realizar un doble esfuerzo. Se debe tener en cuenta que a la altura de 1936 buena parte de las viviendas de la capital contaban con agua corriente pues, aunque «en vísperas de la Guerra Civil española, para la mayoría de familias con ingresos modestos era un lujo contar con una bañera o una ducha dentro de sus viviendas» ${ }^{9}$.

La mejora de los niveles de vida, particularmente en los años posteriores al fin de la Primera Guerra Mundial, incluyeron también la aspiración de que toda familia pudiera contar en su casa con un grifo de cocina y un inodoro. Una aspiración que progresivamente se fue transformando en realidad para un número creciente de habitantes de la ciudad ${ }^{10}$.

Para conseguir alcanzar los objetivos marcados, el escrito se divide en varios apartados. En primer lugar se expone el contexto en el que se va a producir el desabastecimiento de agua, destacando algunas cuestiones clave sobre la huelga general de la construcción; a continuación, siguiendo la prensa de la época, se abordará la cuestión referente al desabastecimiento de agua en sí; por último, se expondrán las conclusiones alcanzadas.

\section{2 - Madrid 1936: la huelga de la construcción de junio-julio/agosto}

En este apartado no se va a exponer la huelga de la construcción de manera extensa, sino que se van a apuntar una serie de cuestiones sobre el contexto en el que

7 “Los peligros de la falta de agua”, El Sol, 9 de julio de 1936, 3 y “¿Para qué?”, ABC, 4 de julio de 1936, 35 .

8 La huelga de los ascensoristas debió iniciarse a principios de mayo ("A consecuencia de una huelga, los ascensores de Madrid no funcionan", La Vanguardia, 23 de mayo de 1936, 25 y “A los obreros de provincias de calefacción y ascensores", El Socialista, 6 de mayo de 1936, 4) y no finalizó hasta el 20 de julio ("Los obreros de calefacción y ascensores se reintegrarán el lunes al trabajo”, $A B C, 18$ de julio de 1936, 24).

$9 \quad$ Nuria Rodríguez Martín, “La capital de un sueño: Madrid 1900-1936: la formación de una metrópoli europea" (Tesis doctoral, Universidad Complutense de Madrid, 2013), 280.

10 Rodríguez Martín, "La capital de un sueño”, 279. A pesar de lo señalado, en julio de 1936 «al extrarradio de Madrid no ha llegado todavía el agua del Lozoya de forma regular», algo que reclamaban sus vecinos, como también lo reclamaban las «zonas de la capital deficientemente dotadas» ("Una nota de Canales del Lozoya. El agua en el extrarradio, El Sol, 7 de julio de 1936,6). Canales del Lozoya es el nombre que adoptó el Canal de Isabel II durante la Segunda República (Rodríguez Martín, "La capital de un sueño”, 261). 
se va a desarrollar el conflicto y sobre la huelga en sí que son fundamentales para entenderlo $^{11}$. Lo primero a destacar es que con la victoria por mayoría absoluta, tanto en Madrid como en el resto de España, del Frente Popular en las elecciones de febrero de $1936^{12}$, y la posterior dimisión del Presidente del Consejo de Ministros, Manuel Portela Valladares, se abre una etapa que permite poner en marcha el programa electoral frentepopulista bajo la dirección de un Gobierno integrado por republicanos de izquierda ${ }^{13}$. Algunas de las medidas contempladas en el acuerdo eran el inicio de un programa de obras públicas encaminado a mitigar el drama del paro, la amnistía y la reincorporación de los trabajadores despedidos (en el caso de la empresa privada se estaría a lo dispuesto por los jurados mixtos aplicando la normativa anterior a noviembre de 1933). Desde el primer día los obreros conscientes salieron a la calle reclamando la aplicación de estos y otros puntos contemplados en el pacto electoral ${ }^{14}$.

Algunas de estas medidas llegaron de forma rápida, pero otras se retrasaron más en el tiempo. En lo que respecta al plano laboral, no se retornó a la legislación de noviembre de 1931 hasta el 2 de junio, siendo cesados los presidentes de los jurados mixtos, lo que obligó a convocar jurados mixtos circunstanciales para resolver los conflictos en marcha, como el de la construcción en Madrid ${ }^{15}$. Esta tardanza en la aplicación de la legislación del primer bienio motivó que muchos de los conflictos laborales se plantearan al margen de la legalidad, recurriendo a la táctica clásica anarcosindicalista de la acción directa ${ }^{16}$, tal y como ocurrió en la huelga madrileña de la construcción.

En este ambiente se iniciaron grandes movilizaciones obreras en la ciudad de Madrid a finales de abril-principios de mayo, convirtiéndose la capital de la República en un hervidero de huelgas con más de 100.000 trabajadores sin acudir a sus empleos ${ }^{17}$, viviendo Madrid «la huelga más general de todas las huelgas generales hasta entonces habidas» ${ }^{18}$.

11 Para conocer en profundidad la huelga de la construcción madrileña en la primavera de 1936: Sánchez Pérez, "La huelga de la construcción en Madrid”, 21-26 y Juliá Díaz, ¿Feudo de la UGT o capital confederal?”, 207-222.

12 Eduardo González Calleja y Francisco Sánchez Pérez, "Revisando el revisionismo. A propósito del libro 1936. Fraude y violencia en las elecciones del Frente Popular”, Historia Contemporánea 58 (2018): 876878.

13 El acuerdo electoral se puede consultar en "El pacto electoral de los partidos de izquierda", El Socialista, 16 de enero de 1936, 1.

14 Sandra Souto Kustrín, «Y ¿Madrid? ¿Qué hace Madrid?»: movimiento revolucionario y acción colectiva (1933-1936) (Madrid: Siglo Veintiuno, 2004), 390-391 y Francisco Sánchez Pérez, "Movilización sociolaboral y oportunidades políticas en España y Francia durante la primavera de 1936", Nuestra Historia: revista de Historia de la FIM 1 (2016): 32.

15 Sánchez Pérez, "Movilización sociolaboral y oportunidades políticas”, 37.

16 Sánchez Pérez, "Clase obrera y conflictividad social”, 57-58.

17 Sánchez Pérez, "Clase obrera y conflictividad social”, 63, aunque el autor no cita la fuente, pero es coincidente con la aparecida en "La situación social derivada", 28, donde se contabiliza el número de obreros en huelga en la ciudad de Madrid por sectores.

18 Santos Juliá Díaz, "Madrid, capital del Estado (1833-1933)”, en: Madrid. Historia de una capital, 
Es en este contexto, con un Madrid inmerso en una oleada de huelgas, en el que se va a desarrollar el conflicto en el ramo de la construcción, iniciado el 1 de junio de 1936 y no finalizado hasta principios del mes de agosto, ya en un contexto completamente diferente a consecuencia del éxito/fracaso parcial del golpe de Estado del 17-18 de julio de 1936.

Para entender el por qué se llega a la huelga general de la construcción en la ciudad de Madrid y las repercusiones que tiene el paro obrero en estos oficios, hay que tener en cuenta que este sector era el que más mano de obra absorbía en los momentos anteriores al estallido de la guerra civil española ${ }^{19}$, exceptuando el servicio domésti$\mathrm{co}^{20}, \mathrm{y}$, de este, dependían otros sectores como los que producían materiales para las obras. No es de extrañar, por tanto, que una huelga en este sector influyera enormemente en la economía de la ciudad, arrastrando por inercia a otros directamente vinculados con la actividad constructora, así como a sectores que en principio nada tenían que ver con ella, como las tabernas o los espectáculos públicos. Además era un sector altamente inestable para el trabajo, pues a la tradicional pérdida de jornales a causa de las inclemencias atmosféricas ${ }^{21}$, se sumaba la posibilidad del aumento del paro, que ya rondaba un tercio de todos los trabajadores del sector ${ }^{22}$, debido al recelo de la patronal, que reducía sus inversiones en este campo cuando la izquierda accedía al poder ${ }^{23}$. Ante esta situación, los obreros defendieron con insistencia el reparto de trabajo a través de la reducción de la jornada laboral (menos horas equivalían a más obreros contratados para poder hacer el mismo trabajo en igual lapso de tiempo), principal argumento a la hora de convocar la huelga de la construcción. Tal y como defendió Edmundo Domínguez, secretario de la ugetista Federación Local de la Edificación (FLE).

Nuestra petición se inspira en que la industria de la edificación, algunos de cuyos oficios, por su fácil acceso a los mismos produce una endémica crisis de trabajo, y de todas las profesiones y de todos los oficios afluyen y disputan el trabajo de las obras públicas, del peonaje en general, creando una situación de competencia, de jornales y

\footnotetext{
Santos Juliá Díaz, David R. Ringrose y Cristina Segura Graíño (Madrid: Alianza Editorial, 2006), 499. Abunda la bibliografía sobre la conflictividad sociolaboral madrileña en la primavera de 1936. Para ampliar sobre esta cuestión: Sánchez Pérez, "Clase obrera y conflictividad social", 47-71; Francisco Sánchez Pérez, "Madrid, capital de la protesta: de agosto de 1917 a julio de 1936", Cuadernos de Historia Contemporánea $\mathrm{N}^{\circ}$ Extra 1 (2007), 301-311; Sánchez Pérez, "Madrid de preguerra, Madrid en huelga. ¿Vísperas de una revolución?", en 1936-1939, la Guerra Civil española: congreso internacional (Madrid 27, 28 y 29 de noviembre de 2006) (Madrid: Sociedad Estatal de Conmemoraciones Culturales, 2008): sin paginar; Francisco Sánchez Pérez, "Las protestas del trabajo en la primavera de 1936", Mélanges de la Casa de Velázquez 41-1 (2011): 77-101; Vadillo, "Entre el pacto y la revolución", 48-64.

19 Julio Montero Díaz y Javier Cervera Gil, "Madrid en los años treinta. Ambiente social, político, cultural y religioso", Studia et Documenta: revista dell'Istituto Storico San Josemaría Escrivá 3 (2009): 20.

20 Juliá Díaz, “¿Feudo de la UGT o capital confederal?”, 212.

21 Sánchez Pérez, "La huelga de la construcción en Madrid", 22.

22 Sánchez Pérez, "Clase obrera y conflictividad social", 51.

23 Sánchez Pérez, "La huelga de la construcción en Madrid", 21.
} 
de miseria, que es preciso cortar con fórmulas tan eficaces, aunque parezcan excesivas, como es la aplicación de la jornada de treinta y seis horas. ${ }^{24}$

Además de la reducción de la jornada laboral de 44 horas ${ }^{25}$ a 36 horas semanales, los obreros defendieron importantes aumentos salariales que iban encaminados a conseguir el fin progresivo de las diferencias entre los distintos oficios, «como paso previo en el fin de las clases y jerarquías» ${ }^{26}$.

Todo partió de una iniciativa de los albañiles y peones de la FLE, quienes en abril, tras aprobar la jornada de 36 horas semanales, invitaron al Sindicato Único de la Construcción (SUC), afectó a la Confederación Nacional del Trabajo (CNT), a elaborar conjuntamente unas nuevas bases de trabajo ${ }^{27}$. Tras diversas reuniones y asambleas, finalmente las nuevas bases de trabajo quedaron aprobadas en una asamblea magna en la Plaza de Toros de las Ventas ante 20.000 obreros $^{28}$ (60.000 según Cipriano Mera ${ }^{29}$, acordándose que se rechazaban los cauces legales de acudir al Jurado mixto, aceptando únicamente la negociación directa con los patronos ${ }^{30}$, lo que significaba adoptar la clásica táctica del anarcosindicalismo.

En esta situación, la huelga estalló el día 1 de junio de 1936, afectando a más de 80.000 obreros, tanto en la ciudad de Madrid como en los municipios próximos, arrastrando desde el comienzo a otros sectores vinculados directamente con la construcción y generando conflictos en el seno de la Unión General de Trabajadores (UGT). Al tiempo que se buscaba causar el mayor número de molestias posibles para forzar a la patronal y al Gobierno, el extrarradio madrileño (Ventas y Cuatro Caminos, lugares que albergaron las asambleas antes y durante la huelga, pero también Puente de Vallecas y las zonas próximas a las carreteras de Valencia y Aragón) se convirtieron en una permanente asamblea de trabajadores en huelga ${ }^{31}$.

La huelga pasó por diferentes etapas, la primera de ellas caracterizada por la unanimidad de acción entre la FLE y el SUC, pero pronto la organización ugetista se desmarcó cuando decidió acudir al Jurado mixto circunstancial a negociar con los patronos, lo que despertó las críticas de los cenetistas. Aunque en el Jurado mixto se aprobaron íntegramente las demandas obreras, un laudo ministerial publicado

$24 \quad$ "Obreros y patronos exponen sus puntos de vista sobre las nuevas bases de trabajo de la construcción. Don Antonio Vallejo Álvarez, arquitecto y constructor y D. Edmundo Domínguez, secretario de la Federación Local de la Edificación, hablan a EL SOL", El Sol, 10 de junio de 1936, 4.

25 Las 44 horas semanales de trabajo estaban vigentes desde 1934 en el sector madrileño de la construcción, tal y como recoge Sánchez Pérez, "Madrid de preguerra", 7.

26 Sánchez Pérez, "Clase obrera y conflictividad social", 67.

27 Sánchez Pérez, "Clase obrera y conflictividad social", 67.

28 Sánchez Pérez, "La huelga de la construcción en Madrid", 22.

29 Juliá Díaz, “¿Feudo de la UGT o capital confederal?”, 290.

30 Juliá Díaz, “¿Feudo de la UGT o capital confederal?, 209-210.

31 Sánchez Pérez, "La huelga de la construcción en Madrid”, 23-25. 


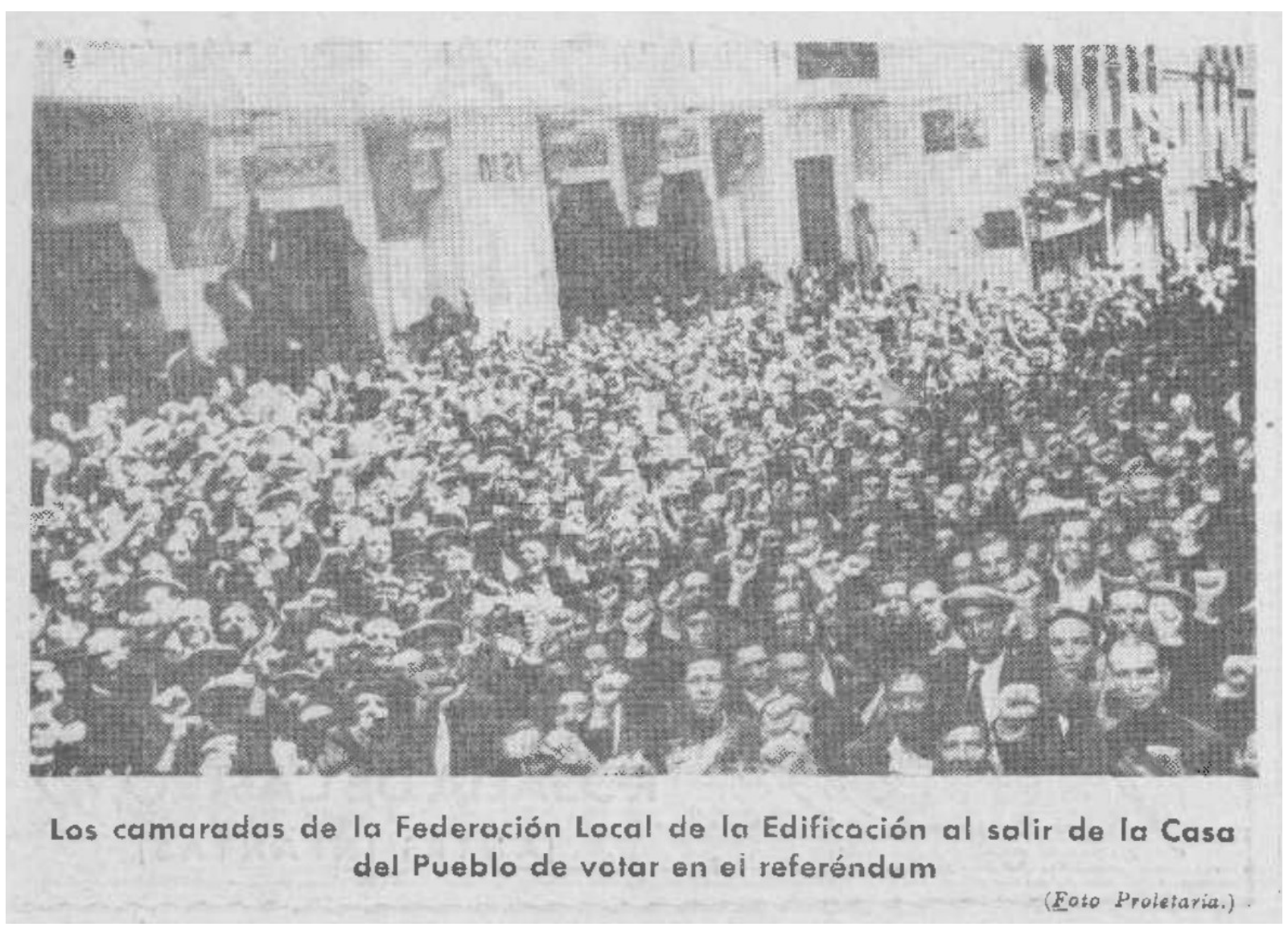

Obreros de la FLE tras votar en el referéndum para aceptar o rechazar acudir al Jurado mixto circunstancial. Fuente: El Socialista, 23 de junio de 1936, 1.

por el Gobierno, tras una reunión en el Consejo de Ministros, rebajó las pretensiones de los obreros ${ }^{32}$.

A partir de aquí se produce un tira y afloja entre la FLE y el SUC y entre albañiles ugetistas y sus compañeros de sindicato, pues los albañiles eran partidarios de mantener la unidad de acción con los anarcosindicalistas, llegando a acordar en asamblea no reintegrarse al trabajo hasta que también lo hicieran sus compañeros del SUC $^{33}$.

32 Las bases que ratificó la asamblea magna de la Plaza de Toros de las Ventas se pueden consultar en "Nuevas bases de trabajo para la Construcción”, La Edificación, 15 de mayo de 1936, 4 y las bases de trabajo establecidas por el laudo ministerial en "El Ministerio de Trabajo dictó ayer el laudo que pondrá fin al conflicto del ramo de la Construcción", Ahora, 4 de julio de 1936, 5 y 11. Sobre la reunión del Consejo de Ministros ver "La huelga de la construcción en Madrid", La Vanguardia, 4 de julio de 1936, 20.

33 "Elementos de la U. G. T. acuerdan no volver al trabajo en tanto no lo hagan los de la C. N. T.", $L a$ Vanguardia, 12 de julio de 1936, 21. 
Aún con la huelga sin solucionar, estallaba en España una guerra civil tras el fracaso/éxito parcial del golpe de Estado del 17-18 de julio. La solución al conflicto acabaría llegando poco después, ya en un contexto completamente diferente a cuando se inició, tras la emisión de una Orden por parte del Gobierno obligando a los obreros de la construcción que aún estaban en huelga a reintegrarse al trabajo el día 3 de agosto $^{34}$, cosa que parece que se cumplió, tal y como se recogió en la prensa:

Ha bastado una orden para que los obreros del ramo de la construcción de Madrid, que se encontraban en huelga desde hacía tiempo, se reintegrasen al trabajo, prescindiendo de toda diferencia, con un magnífico espíritu de disciplina y un alto sentido de orden ${ }^{35}$

\section{3 - El desabastecimiento de agua en la ciudad de Madrid}

\section{1 - Las zonas afectadas}

Se hace prácticamente imposible establecer, a través de las fuentes hemerográficas, el alcance real del desabastecimiento de agua en estas semanas de 1936, aunque sí se pueden definir algunas coordenadas de las averías. Con el paso de los días, algún periódico llegó a afirmar que los cortes afectaron a «la mayoría de los distritos de la ciudad $»^{36}$ y «a la inmensa mayoría de los madrileños» ${ }^{37}$, lo que sin duda es una exageración, aunque las zonas afectadas fueron numerosas. Desde los primeros días de la huelga general de la construcción algunas zonas de Madrid quedaron privadas de agua debido a que el Comité conjunto que dirigía el conflicto, formado por miembros del SUC y de la FLE, dio la orden de paralizar «todas las reparaciones que se hacían en la calle de agua $\aleph^{38}$. Quedaron así sin agua distintas calles del distrito de Hospicio, como «las calles de Larra, Apodaca y las inmediaciones» ${ }^{39}$ (posiblemente ese «inmediaciones» se refiere a «la populosa calle de la Florida y adyacentes» que también sufrieron los cortes) $)^{40}$. Con el paso de los días (hacia el 17-18 de junio) estos cortes se exten-

34 Ministerio de Trabajo, Sanidad y Previsión, “Orden disponiendo que los obreros de la construcción se reintegren al trabajo en todas las obras, tajos, fábricas y talleres, en que estuvieren ocupados, el próximo lunes 3", Gaceta de Madrid, 2 de agosto de 1936, 1002.

35 La Vanguardia, 6 de agosto de 1936 (Suplemento), portada

36 "Mientras en Madrid se carece de agua y de otras cosas, los ediles se dedican a hablar del Papa", $L a$ Voz, 3 de julio de 1936, 6 .

37 "Todos son celosos y enérgicos...; pero el agua no aparece. Se impone con urgencia la necesidad de denunciar el Convenio con Canales del Lozoya", La Voz, 4 de julio de 1936, contraportada.

38 "Estado de la huelga de la construcción", $A B C, 7$ de junio de 1936, 47.

39 "El suministro de agua en el barrio de Bilbao", La Voz, 2 de julio de 1936, 1.

$40 \quad$ "Un barrio madrileño sin agua", Ahora, 17 de junio de 1936, 21. 
dieron a las calles Núñez de Balboa y Goya y otras zonas del barrio de Salamanca ${ }^{41}$. Un detallado registro de las áreas afectadas se encuentra en la revista Crónica, siendo estos lugares «las calles de Churruca, Larra, Barceló, Hermosilla, Carranza, parte de la Castellana, parte de la calle de Toledo, Puente de Toledo, barrio de Cuchilleros y Puerta Cerrada, barriada del Puente de Segovia, Plaza de España, barrio de Chamberí $\rangle^{42}$, aunque no se puede descartar que alguna otra zona también se viera afectada. En algún caso se puede incluso concretar el número de la calle que quedó sin agua, como es el caso de la calle Segovia, $\mathrm{n}^{\circ}$ 4, donde se vio afectado por los cortes el Dispensario Azúa, que debió cerrar ${ }^{43}$. Nadie se libró de los cortes de agua, ni siquiera los edificios oficiales extranjeros, pues hasta una Embajada, situada en el paseo de la Castellana, sufrió este inconveniente y debió ser abastecida mediante tanques enviados por el Ayuntamiento ${ }^{44}$.

\section{2 - Los más humildes, los más afectados}

Por lo general se va a considerar que los más afectados por los cortes de agua son los ciudadanos más humildes de Madrid, «pobres mujeres de hogares humildes, niños mal vestidos, ancianos, sirvientas... Todos pertenecientes a una clase modestísima $\rangle^{45}$, mientras que las personas pudientes no se ven afectadas por la falta de tan preciado líquido en sus viviendas ${ }^{46}$ (es de suponer que esto se debe a que tenían personal doméstico en sus casas que se encargaría de ir a recoger el agua). Que a quienes se estaba perjudicando era «precisamente a quienes, pensando lógicamente, menos interés debían tener los obreros en perjudicar ${ }^{47}$ parece una realidad, dado que desde la prensa socialista se apuntó que, para ganarse las simpatías de los ciudadanos de Madrid, era necesario «acabar con aquellas molestias que al vecindario ocasiona la falta de agua en determinadas zonas», pues además la situación creada era usada «por los periódicos adversos a la posición obrera» ${ }^{48}$.Incluso la prensa socialista llegó a usar

$41 \quad$ "Vecinos del barrio de Salamanca sin agua", $A B C, 20$ de junio de 1936, 29.

42 R. M. G., "El lado pintoresco de las huelgas. La "cola" del agua, los tanques del Ayuntamiento y las cañerías rotas", Crónica, 5 de julio de 1936, 12.

43 "Madrid en la cruz", $A B C, 9$ de julio de 1936, 9.

44 "Todos son celosos", contraportada.

45 R. M. G., "El lado pintoresco de las huelgas", 12. Otras referencias donde se destaca que son este tipo de gentes quienes se ven afectadas en "El suministro de agua", 1 y Ángel Ossorio, "La fuente y la 'cola", Ahora, 30 de junio de 1936, 5.

46 Ossorio, "La fuente", 5.

47 R. M. G., "El lado pintoresco de las huelgas", 12.

48 "La huelga de la construcción. La retirada de los patronos descubre sus intenciones", El Socialista, 24 de junio de 1936, 1. Este artículo sería usado por "Un cambio de táctica", El Sol, 25 de junio de 1936, 1 para atacar la actitud de los obreros en huelga, al tiempo que les recomendaba hacer caso al órgano oficial del Partido Socialista Obrero Español (PSOE). En "El suministro de agua", 1 también se va a apuntar que los huelguistas 


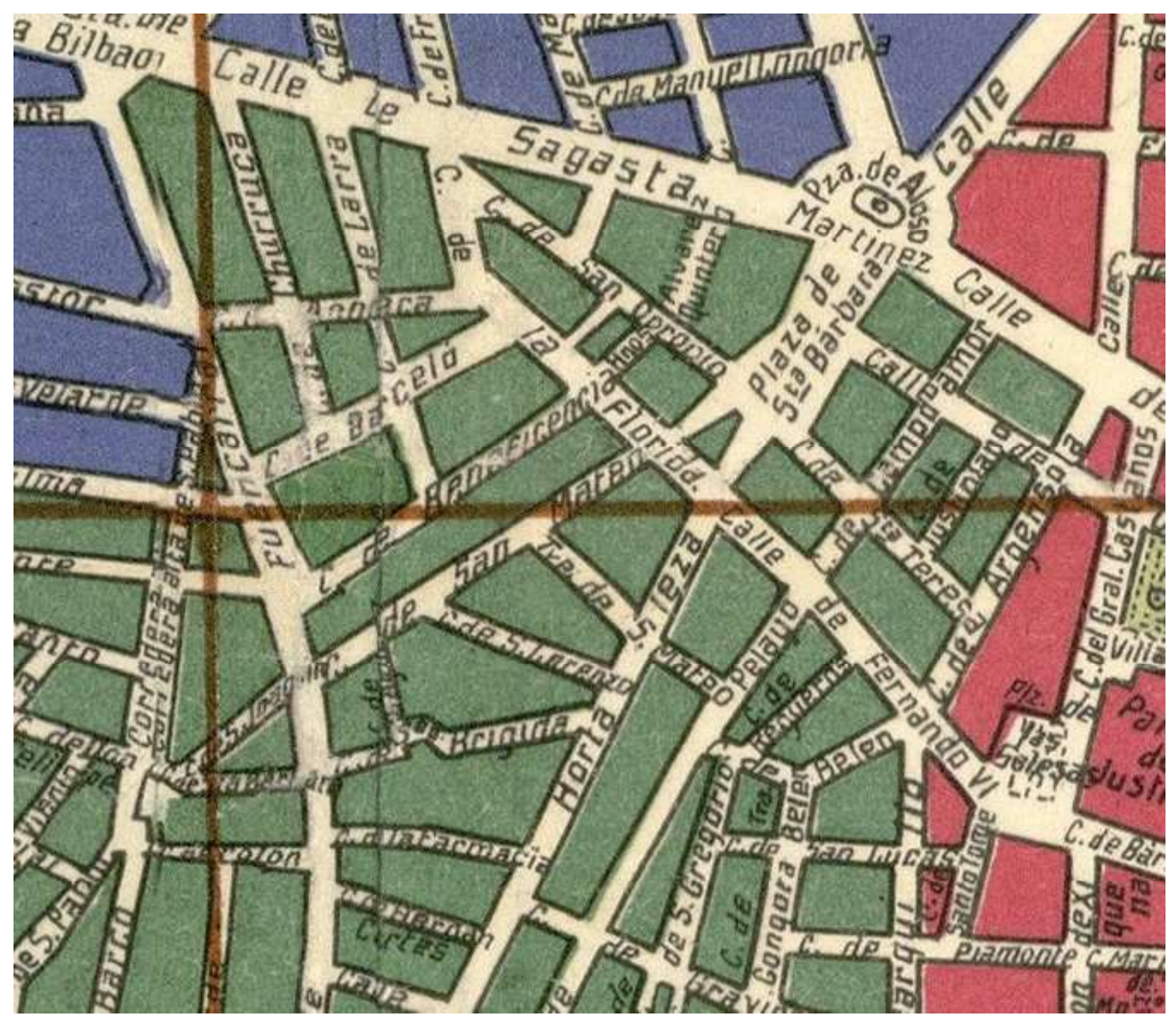

Detalle de un plano de Madrid del año 1934 donde se muestran algunas de las calles afectadas por los cortes de agua (calles de Larra,

de la Florida, Churruca, Barceló) en el distrito de Hospicio.

Fuente: Sección de Estadística del Ayuntamiento de Madrid, Madrid: plano de sus vías, Madrid: Imprenta de las Artes Gráficas Municipales, 1934.

esta cuestion como argumento para atacar la posición del SUC de rechazar el laudo ministerial, asegurando que los anarcosindicalistas no pueden obtener la victoria siguiendo el método de la acción directa por tres cuestiones, una de ellas «el desafecto de la opinión, desafecto que tiene su origen no en la huelga y sí en sus derivaciones enojosas, siendo la más grave la que ha dejado sin agua a una parte del vecindario» ${ }^{49}$.

harían bien en permitir las reparaciones para conseguir así atraerse las simpatías de los afectados y en "La huelga y la falta de agua", El Sol, 20 de junio de 1936, 3 se va a considerar que al no permitirse efectuar las reparaciones los obreros lo que hacen es ganarse la enemistad de los vecinos.

49 "Referéndum y Asamblea. El desenlace de la huelga de la Construcción”, El Socialista, 7 de julio de 
Aunque la posición del órgano del PSOE hay que entenderla en su contexto, es decir, una vez rota la unidad de acción entre la FLE y el SUC, primero decidiendo los ugetistas acudir al Jurado mixto circunstancial y luego dando el visto bueno al laudo ministerial que fijaba las nuevas bases de trabajo, esto evidencia que buena parte de las clases populares madrileñas veían cada vez con más recelo a los huelguistas de la construcción, quienes se oponían a que se llevaran a cabo las reparaciones de las averías en la red suministradora de agua.

\section{3 - ¿Solidaridad o coacción?}

Por un motivo u otro, la realidad era que los obreros de Canales del Lozoya no reparaban las averías ${ }^{50}$, aunque desde la prensa se argumentaba que permitir las reparaciones no hubiera afectado en nada a la huelga de la construcción ${ }^{51}$, opinión que obviamente no compartían los obreros, quienes consideraban que cuantas más molestias se causasen, antes se forzaría a la patronal y al Gobierno. Incluso los obreros ugetistas de la Sociedad de Trabajadores de Agua, Gas y Electricidad se quejaron de esta situación por medio de un escrito publicado en la prensa y firmado por su secretario, Ricardo Rouco (la fecha de la nota varía dependiendo la fuente, para $E l S o l$ y $A B C$ es el 18 de junio, para El Socialista el 19 de junio) ${ }^{52}$. Van a negar que ellos estén secundando los paros, asegurando que han intentado que se les autorice reparar las averías, sin conseguirlo, y que cuando han iniciado algún trabajo han debido parar, pues para evitar enfrentamientos tienen orden de retirarse. El final de la nota indica que los piquetes protagonizados por los huelguistas (en algunos casos debió intervenir la Guardia de Asalto $)^{53}$ para impedir los trabajos no debían ser precisamente pacíficos, pues

no existe por parte de los trabajadores de Agua, Gas y Electricidad, ningún deseo de obstaculizar la marcha de la capital de la República, y sí tan sólo interés en que su suelo no quede regado por la sangre de nuestros asociados ${ }^{54}$.

La revista Crónica, publicación que en las líneas anteriores a las que aquí se van a recoger pretende dar un cierto toque de humor ${ }^{55}$ a la situación al reproducir una

$1936,1$.

50 R. M. G., "El lado pintoresco de las huelgas", 12. Para Sánchez Pérez, "Clase obrera y conflictividad social", 68 se trata de una solidaridad impuesta fruto de la coacción.

51 "El suministro de agua", 1.

52 "La huelga y la falta", 3, "Agua, gas y electricidad no se han sumado a la huelga de la construcción", $A B C, 20$ de junio de 1936, 31 y "La huelga de la Construcción parece condenada a eternizarse en el mismo estado en que se planteó el primer día", El Socialista, 20 de junio de 1936, 5.

53 "El suministro de agua", 1.

54 "La huelga y la falta", 3 y "La huelga de la Construcción parece condenada", 5.

55 Otros ejemplos del toque de humor que se le quiso dar al desabastecimiento del agua es una viñeta 
supuesta conversación en una de las colas formadas para recoger agua, va a apuntar en esta misma dirección, que los obreros de Canales del Lozoya eran partidarios de reparar las averías (y, por tanto, que no existiría tal solidaridad sino coacción):

Mientras, los obreros del Canal, en los mismos sitios donde se han producido las averías, contemplan las escenas de la «cola» fumando un cigarro.

- ¡Míralos! ¡Vamos, se necesita valor para presentarse aquí!

- ¿Y qué culpa tenemos nosotros? ¡Si estamos deseando arreglarlo!

Y eso es lo curioso. Todos están deseando arreglarlo y no se arregla. La vida ofrece estos misterios indescifrables ${ }^{56}$.

LAS COLAS DEL AGUA, por Echea

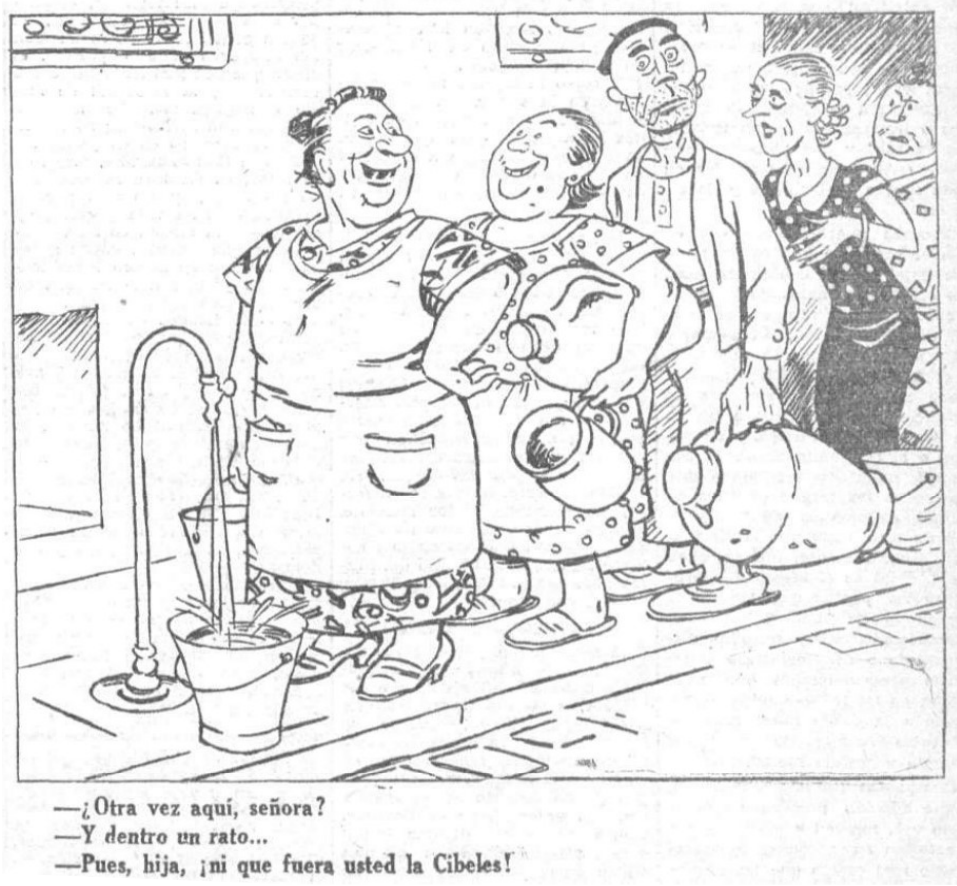

Viñeta donde se muestra un grupo de madrileños haciendo cola frente a un surtidor de agua para abastecerse, pues la no reparación de las averías en la red suministradora, a consecuencia de la huelga de la construcción, les obligaba a ello. Fuente: La Voz, 7 de julio de 1936, 1.

Parece que se llegaron a cometer actos de sabotaje en las conducciones de agua en la ciudad de Madrid e, incluso, en las fuentes que abastecían a los vecinos privados

publicada en $L a$ Voz, 7 de julio de 1936, 1 y el artículo «El caso es pegar», Ahora, 9 de julio de 1936, 7.

56 R. M. G., "El lado pintoresco de las huelgas", 12. 
de tan preciado líquido ${ }^{57}$, aunque la prensa no especificaba que estuvieran vinculados estos actos con la huelga de la construcción ni protagonizados por sus obreros ${ }^{58}(\operatorname{cosa}$ que no se puede descartar), era algo que sí parecía tener claro el diputado Antonio Bermúdez Cañete. Si la huelga de la construcción en Madrid había sido un conflicto abordado en las más altas esferas del poder del Estado ${ }^{59}$, no lo sería menos la falta de agua en la capital de la República, saliendo a colocación en los debates en la residencia del poder legislativo. Si el diputado Ramón Suárez Picallo usaba esta cuestión a modo de burla («Le advierto al Sr. [Jesís] Comín [Sagües] que desde hoy funcionan maravillosamente los Canales del Lozoya, bebida riquísima, sin consecuencias dialécticas») ${ }^{60}$, el diputado Bermúdez Cañete recurriría a la falta de agua en Madrid para atacar al Gobierno:

[...] Sin duda cree el Gobierno, como luego veremos, que los madrileños beben más agua cuando no leen la prensa que cuando notan que en su casa ya no existe ese elemento.

\section{$[\ldots]$}

Es evidente que estas huelgas están produciendo, en concatenación de causa a efecto, una serie de trastornos no ya de orden público, sino materiales. Esto se halla a la vista de todos. Las cañerías saltan por sabotaje; [...] y actualmente se da el caso lamentable de que el Sanatorio de San José, en el cual asisten a nuestras familias de los socios de la Asociación de la Prensa, lleva exactamente una semana sin agua y sin electricidad. Es decir, en el sanatorio de los periodistas madrileños, que se supone serían los más divulgadores de las bondades del actual régimen y del Gobierno, hace una semana $[\ldots]$ que las monjitas tienen que lavar con sus manos la cantidad tan enorme de ropa que necesita un sanatorio, porque, como digo, no hay agua por conducción directa

57 "Dejan sin agua a los vecinos de las calles de Larra y Churruca", $A B C, 17$ de junio de 1936, 39.

58 "Actos de sabotaje en las conducciones de agua", El Sol, 8 de julio de 1936, 4 y "Anoche fueron producidas averías de consideración en dos importantes conducciones de agua de Canales del Lozoya", El Socialista, 8 de julio de 1936, 1.

59 Los diputados De Francisco (socialista) y José Díaz (comunista) debieron intervenir ante el Gobierno por la posibilidad de que el laudo ministerial que fijaba las bases de trabajo quedara sin efecto ante la no reincorporación de los obreros al trabajo, ya fuera por voluntad propia o por presiones (pacíficas o violentas) de aquellos partidarios de mantener la huelga. Los dos diputados consiguieron reunir en una conferencia a directivos de la FLE y a buena parte de los miembros del Gobierno, incluyendo al presidente Casares Quiroga, de donde salió el compromiso de volver al trabajo el día 13 de julio, lo que suponía prorrogar el tiempo que se les daba a los trabajadores para reintegrarse en las obras para que no quedaran sin efecto las nuevas bases de trabajo ("La tramitación del conflicto político planteado por la huelga de la construcción", La Vanguardia, 11 de julio de 1936, 21). Además, la huelga de la construcción en Madrid fue usada por el diputado José María Gil-Robles como argumento en sus ataques al Gobierno, destacando la alta violencia que envolvía al conflicto, pintando un Madrid al borde del caos y considerando que las organizaciones obreras estaban cercenando la autoridad gubernamental (Sesión de la Diputación Permanente de Cortes, sesión del miércoles 15 de julio de $1936,6-7)$.

60 Congreso de los Diputados, Diario de las Sesiones de Cortes, 58, 8 de julio de 1936, 1986. 
ni hay fuerza motriz eléctrica para elevarla a los pisos superiores.

$[\ldots]$

Nosotros hace exactamente una semana hicimos un ruego por escrito, concretísimo, sobre los trastornos en el suministro de agua y de electricidad en Madrid, a los Sres. Ministros de Obras públicas y de Gobernación. El ruego no ha sido publicado, como ya hice constar, en la prensa ${ }^{61}$.

Para intentar amortiguar las consecuencias que pudiera tener la falta de agua en los distintos puntos de la ciudad, el Ayuntamiento se dedicó a enviar tanques de agua, cada uno de ellos con una capacidad de $2.500 \operatorname{litros}^{62}$ para surtir a los vecinos, quienes también tenían a su disposición las bocas de riego y las fuentes públicas ${ }^{63}$. Estos tanques municipales en muchas ocasiones no eran suficientes, viéndose los afectados obligados a esperar la llegada de otro si querían llenar sus recipientes ${ }^{64}$. El Ayuntamiento, al parecer, se negaba a dar la orden para que se acometieran las reparaciones de las averías, pues eso significaba mantener una actitud beligerante hacia los huelguistas e incurrir en prácticas de esquiroloje ${ }^{65}$, aunque para algún periódico también era esquirolaje dejar que los vecinos obtuvieran agua a través de los tanques municipales o de las bocas de riego, a la vez que dedicaba el personal de limpieza municipal $a$ atender estar tareas, impidiendo que se pudiera regar la ciudad ${ }^{66}$.

A pesar de los reproches que desde la prensa se hacían al Ayuntamiento por su negativa a obligar las reparaciones, esta no parecía tener muy claro a quién correspondía tomar la iniciativa en el arreglo de las conducciones, por lo que repartía sus críticas entre el Ayuntamiento, Canales del Lozoya (quienes a su vez se acusaban mutuamente) ${ }^{67}$ y el Estado, pues a él pertenecía el servicio de abastecimiento de agua y era quien «tiene la obligación de asegurarlo, sin que puedan valer de pretexto para el incumplimiento de su deber las luchas sociales que se registran ${ }^{68}$. El no saber quién era competente para obligar a hacer las reparaciones posiblemente se deba al hecho de que en la ciudad de Madrid la red de distribución de agua tenía distintos titulares, siendo estos el Ayuntamiento, Canales del Lozoya (el Estado) y algunos particulares ${ }^{69}$, aunque se puede afirmar que la obligación era en exclusiva de Canales del Lozoya, no solo porque fueron sus obreros quienes acabaron reparando las averías, sino porque el concejal Cort (monárquico y, por tanto, poco sospechoso de simpatizar con el Gobier

61 Congreso de los Diputados, Diario de las Sesiones de Cortes, 58, 8 de julio de 1936, 1990-1991.

62 R. M. G., "El lado pintoresco de las huelgas", 12.

63 Un barrio madrileño sin", 21 y "Mientras en Madrid se carece", 6.

$64 \quad$ H., «Feria de ingenuos», El Sol, 18 de junio de 1936, contraportada.

65 H., "Feria", contraportada.

66 "Mientras en Madrid se carece", 6.

67 "Todos son celosos", contraportada.

68 "La huelga y la falta", 3.

69 Rodríguez Marín, "La capital de un sueño", 268. 


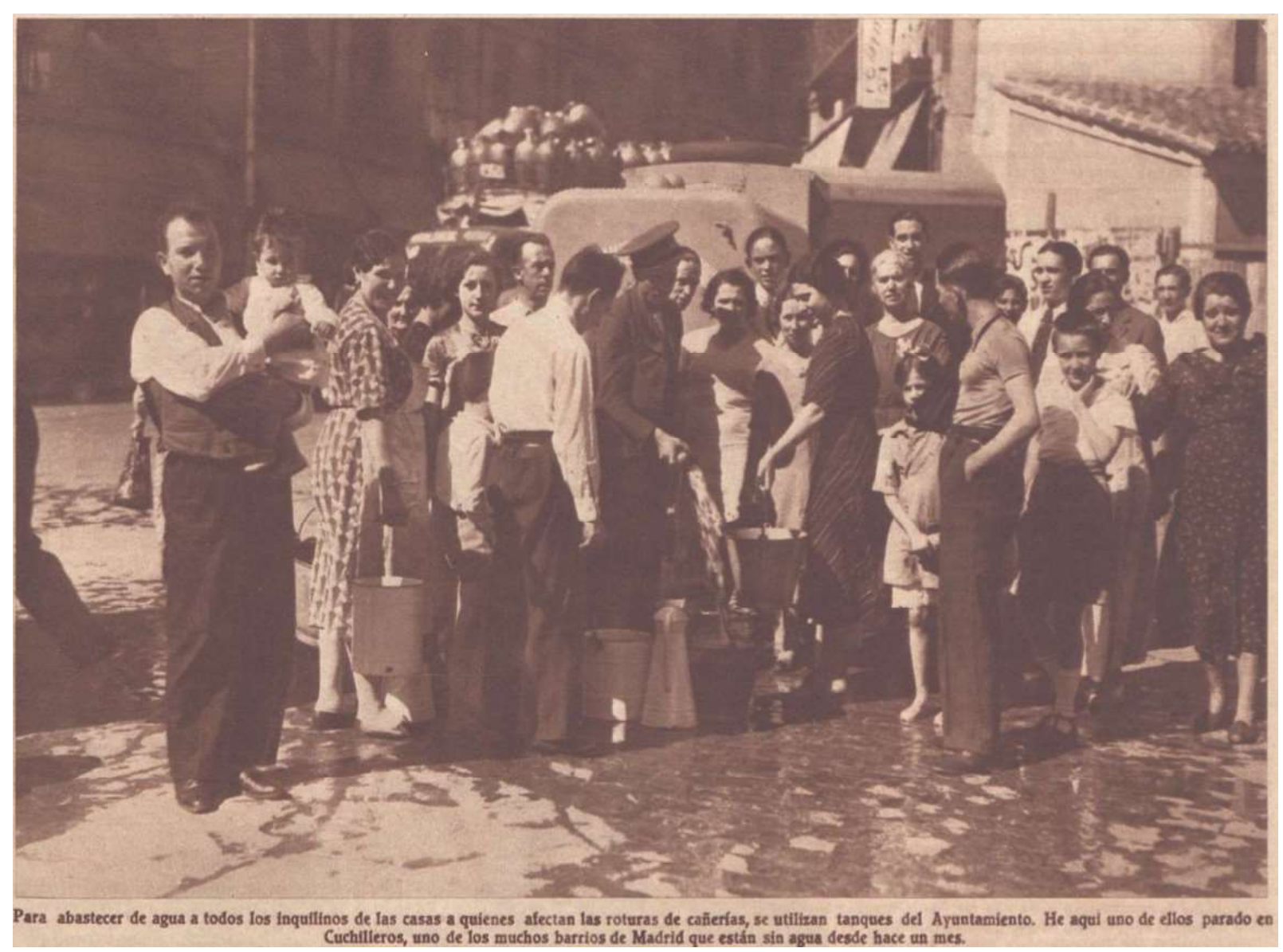

Vecinos de Cuchilleros frente a un tanque municipal esperando abastecerse de agua. En esos días debió ser una imagen común en muchos puntos de Madrid. Fuente: R. M. G., «El lado pintoresco de las huelgas. La "cola” del agua...», 11.

no municipal o el Gobierno de España) intentó en el pleno del Ayuntamiento, sin éxito, proponer al consistorio que se solicitara a Canales del Lozoya la reparación de todas las averías y, de no conseguirse, que fuera el Ayuntamiento quien tomara la iniciativa, efectuando las reparaciones el personal municipal y pasando luego la factura a Canales del Lozya ${ }^{70}$.

Finalmente entre los días 8 y 10 de julio se procedió a la reparación de las diversas averías, quedando restablecida buena parte del servicio ${ }^{71}$ y destacándose la

70 "Intereses de Madrid. Las subvenciones a los centros obreros y las obras en el camino de Leñeros. El Ayuntamiento concede una cantidad para la construcción del monumento al periodista Luis de Sirval", El Sol, 4 de julio de 1936, 4.

71 "A partir de media tarde ha empezado a tener agua Madrid", La Voz, 8 de julio de 1936, contraportada; "Se restablece el abastecimiento de agua que faltaba en algunos sectores", Ahora, 9 de julio de 1936, 21; "Ayer quedó restablecida la normalidad en el abastecimiento de agua", Ahora, 10 de julio de 1936, 21; y "Una nota de 
celeridad con que se estaban empeñando los trabajadores de Canales del Lozoya ${ }^{72}$, siendo la zona de la calle de Larra la última en recobrar la normalidad ${ }^{73}$. Madrid volvía a tener agua.

\section{SE ACABARON LAS COLAS}

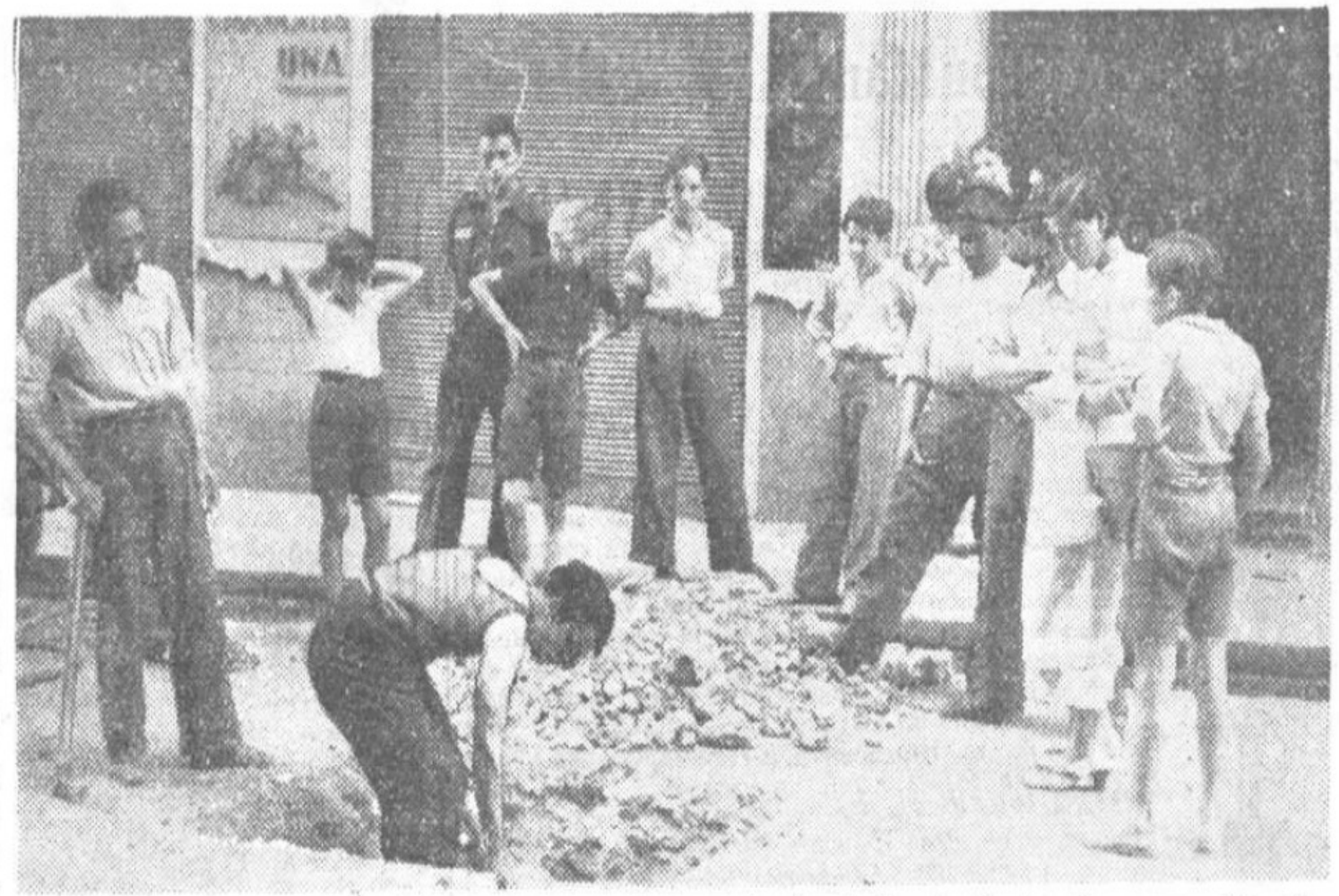

Los obreros de Canales del Lozoya comenzar on esta tarde los trabajos de reparación de las cañerías y fueron rodeados afectuosamente por el público.

(Foto Alfonso.)

Fuente: La Voz, 8 de julio de 1936, 10.

\section{4 - Conclusiones}

La huelga general del ramo de la construcción en Madrid de junio y julio/agosto de 1936 fue un movimiento ofensivo de la clase trabajadora en busca de importantes mejoras laborales que supuso el paro de más de 80.000 obreros y que acabó arrastrando

Canales del Lozoya", El Sol, 10 de julio de 1936, 4.

72 "A partir de media tarde", contraportada.

73 "Reparación de las averías en las conducciones de agua", $A B C, 11$ de julio de 1936, 39. 
a otros sectores con los que estaban vinculados. La huelga, convocada conjuntamente por la FLE y el SUC, se hizo siguiendo la táctica clásica anarcosindicalista de la acción directa, aunque pronto parte de la militancia ugetista abandonó los compromisos adquiridos y accedió a negociar en el marco legal, lo que supuso tanto enfrentamientos entre obreros de uno y otro sindicato como una ruptura en el proceder de la bases de la FLE, enfrentándose albañiles y peones (partidarios a volver a actuar en comunión con el SUC) al resto de la organización. La huelga no se resolvería hasta una vez empezada la guerra civil española tras el fracaso/éxito parcial del golpe de Estado del 17-18 de julio de 1936, ya en un contexto completamente distinto a cuando se inició.

Una huelga de dimensiones tan colosales, en un sector tan importante como el de la construcción y que desde el principio arrastró a otros oficios, debía acabar teniendo efectos sobre la vida cotidiana de los vecinos de Madrid, siendo el más relevante de ellos la falta de agua por la no reparación de las averías en la red suministradora. Calles y barrios de la capital de la República se quedaron sin agua, algunos de ellos durante un mes, a las puertas del verano o ya entrado en él. La vida de una parte de los habitantes de la ciudad se veía alterada, pues el agua no llegaba a sus casas y debían hacer largas colas para abastecerse de tan preciado líquido a través de los tanques municipales, las bocas de riego o las fuentes públicas. Esta anormalidad supondría que, tarde o temprano, la población de Madrid acabara viendo con malos ojos el proceder de los huelguistas del ramo de la construcción, tal y como terminó advirtiendo la prensa.

Ante esta situación el Ayuntamiento de Madrid respondió enviando tanques municipales con agua para abastecer a los vecinos que sufrían las averías, negándose a dar la orden para la reparación de las mismas, ya fuera a Canales del Lozoya o a sus propios empleados, algo que podría haberle enemistado con los obreros, los cuales se suponía que habían votado a los miembros del FP en las elecciones generales de febrero de 1936 y tenían como táctica para alcanzar sus objetivos crear el mayor número de inconvenientes para doblegar así a patronos y Gobierno.

El Comité que dirigía la huelga, formado por representantes de la FLE y del SUC, fue quien dio la orden de no realizar las reparaciones de las averías, por lo que se puede suponer que ambas organizaciones apoyaron esta táctica en un principio. Pero igual que la FLE se acabó desvinculando de lo acordado en la asamblea magna del 19 de mayo en lo referente a llevar la huelga por el camino de la acción directa, acabó rechazando el proceder del Comité de huelga, advirtiendo de los perjuicios que tendría para los obreros continuar por el camino de crear el mayor número de inconvenientes posibles en la ciudad de Madrid, llegando a reprochar la actitud cenetista de no permitir las reparaciones en las conducciones de agua. Para el caso del SUC no se ha podido determinar su posición tras varias semanas de huelga, lo que lleva a la pregunta de por qué permitieron los trabajados de los empleados de Canales del Lozoya a partir del día 
8 de julio. ¿Aceptaron el punto de vista de los ugetistas y de la prensa de que por ese camino no harían otra cosa que poner a los madrileños en su contra? Estas preguntas es posible que se pudieran responder consultando el periódico que editó el SUC en la primavera de 1936, Construcción: órgano del Sindicato Único del Ramo (disponible en la Hemeroteca Municipal de Madrid), que no se ha podido consultar.

Tal y como se ha visto, la prensa dedicó gran atención al desabastecimiento de agua en Madrid, en especial tras poco más de dos semanas de huelga, cuando la situación se hacía cada vez más insoportable para una parte de los madrileños. La mayoría de los periódicos consultados rechazaron el proceder de los huelguistas al no permitir las reparaciones en la red suministradora de agua, destacando que en nada les beneficiaba su actitud, igual que atacaron a la corporación municipal por su pasividad hacia los obreros, aunque no faltaron los toques de humor sobre esta cuestión.

Pero no fueron los periódicos los únicos que prestaron atención a la falta de agua, sino que también la minoría de derechas en el Congreso de los Diputados recurrió a la huelga de la construcción y al desabastecimiento de agua en la capital de la República para atacar al Gobierno y dibujar un Madrid al borde del caos.

\section{Bibliografía}

\section{Fuentes primarias}

\section{Fuentes hemerográficas ${ }^{74}$ (orden cronológico ${ }^{75}$ )}

"El pacto electoral de los partidos de izquierda", El Socialista, 16 de enero de 1936.

"A los obreros de provincias de calefacción y ascensores", El Socialista, 6 de mayo de 1936.

"Nuevas bases de trabajo para la Construcción”, La Edificación, 15 de mayo de 1936.

$74 \quad$ Nota del editor: si bien es cierto que, según las normas de citación, la bibliografía va en orden alfabético, por motivos prácticos para futuras investigaciones sobre el tema, se prefirió dejar las fuentes hemerográficas en orden cronológico. Esto con la intención de hacer más fácil la búsqueda por temporalidades para otros investigadores.

75 Los ejemplares de Ahora, Crónica, El Sol y La Voz disponibles en la Hemeroteca Digital de la Biblioteca Nacional de España. Consultado el día 5 de febrero de 2019, http://hemerotecadigital.bne.es/index.vm; los ejemplares de El Socialista disponibles en la Hemeroteca de la Fundación Pablo Iglesias. Consultado el 5 de febrero de 2019, http://archivo.fpabloiglesias.es/index.php?r=hemeroteca/ElSocialista; el ejemplar de La Edificación disponible en Memoria de Madrid. Consultado el 5 de febrero de 2019, http://www. memoriademadrid.es/buscador.php?accion=VerFicha\&id=38955; el ejemplar de El Liberal (Bilbao) disponible en Liburuklik: Biblioteca Digital Vasca. Consultado el 5 de febrero de 2019, http://www.liburuklik. euskadi.eus/handle/10771/11967. 
"A consecuencia de una huelga, los ascensores de Madrid no funcionan", La Vanguardia, 23 de mayo de 1936.

"Estado de la huelga de la construcción”, ABC, 7 de junio de 1936.

"Obreros y patronos exponen sus puntos de vista sobre las nuevas bases de trabajo de la construcción. Don Antonio Vallejo Álvarez, arquitecto y constructor y D. Edmundo Domínguez, secretario de la Federación Local de la Edificación, hablan a EL SOL”, El Sol, 10 de junio de 1936.

"Dejan sin agua a los vecinos de las calles de Larra y Churruca", ABC, 17 de junio de 1936.

“Un barrio madrileño sin agua”, Ahora, 17 de junio de 1936.

H. «Feria de ingenuos», El Sol, 18 de junio de 1936.

"Ochenta mil trabajadores llevan ya diecisiete días de huelga. Urge que se le ponga remedio", El Socialista, 18 de junio de 1936.

"Agua, gas y electricidad no se han sumado a la huelga de la construcción", ABC, 20 de junio de 1936.

"La huelga de la Construcción parece condenada a eternizarse en el mismo estado en que se planteó el primer día”, El Socialista, 20 de junio de 1936.

“La huelga y la falta de agua", El Sol, 20 de junio de 1936.

"Vecinos del barrio de Salamanca sin agua", ABC, 20 de junio de 1936. El Socialista, 23de junio de 1936.

Prieto, Indalecio. "Enseñanzas de una huelga", El Liberal (Bilbao), 23 de junio de 1936.

"La huelga de la construcción. La retirada de los patronos descubre sus intenciones", El Socialista, 24 de junio de 1936.

“Un cambio de táctica”, El Sol, 25 de junio de 1936. 
“La situación social derivada de las huelgas”, La Vanguardia, 26 de junio de 1936.

Ossorio, Ángel. "La fuente y la "cola", Ahora, 30 de junio de 1936.

"El suministro de agua en el barrio de Bilbao", La Voz, 2 de julio de 1936.

"Mientras en Madrid se carece de agua y de otras cosas, los ediles se dedican a hablar del Papa", La Voz, 3 de julio de 1936.

"El Ministerio de Trabajo dictó ayer el laudo que pondrá fin al conflicto del ramo de la Construcción”, Ahora, 4 de julio de 1936.

"Intereses de Madrid. Las subvenciones a los centros obreros y las obras en el camino de Leñeros. El Ayuntamiento concede una cantidad para la construcción del monumento al periodista Luis de Sirval", El Sol, 4 de julio de 1936.

“La huelga de la construcción en Madrid”, La Vanguardia, 4 de julio de 1936.

“¿Para qué?”, ABC, 4 de julio de 1936.

"Todos son celosos y enérgicos...; pero el agua no aparece. Se impone con urgencia la necesidad de denunciar el Convenio con Canales del Lozoya", La Voz, 4 de julio de 1936.

R. M. G. "El lado pintoresco de las huelgas. La "cola" del agua, los tanques del Ayuntamiento y las cañerías rotas", Crónica, 5 de julio de 1936.

La Voz, 7 de julio de 1936.

"Referéndum y Asamblea. El desenlace de la huelga de la Construcción", El Socialista, 7 de julio de 1936.

“Una nota de Canales del Lozoya. El agua en el extrarradio, El Sol, 7 de julio de 1936.

“Actos de sabotaje en las conducciones de agua", El Sol, 8 de julio de 1936.

"Anoche fueron producidas averías de consideración en dos importantes conducciones de agua de Canales del Lozoya", El Socialista, 8 de julio de 1936.

"A partir de media tarde ha empezado a tener agua Madrid", La Voz, 8 de julio de 
1936.

«El caso es pegar», Ahora, 9 de julio de 1936.

"Los peligros de la falta de agua", El Sol, 9 de julio de 1936.

“Madrid en la cruz", ABC, 9 de julio de 1936.

"Se restablece el abastecimiento de agua que faltaba en algunos sectores", Ahora, 9 de julio de 1936.

"Ayer quedó restablecida la normalidad en el abastecimiento de agua", Ahora, 10 de julio de 1936.

"Una nota de Canales del Lozoya", El Sol, 10 de julio de 1936.

"La tramitación del conflicto político planteado por la huelga de la construcción", La Vanguardia, 11 de julio de 1936.

"Reparación de las averías en las conducciones de agua", ABC, 11 de julio de 1936.

"Elementos de la U. G. T. acuerdan no volver al trabajo en tanto no lo hagan los de la C. N. T.”, La Vanguardia, 12 de julio de 1936.

La huelga de la construcción ha entrado en la séptima semana", La Vanguardia, 15 de julio de 1936.

"Los obreros de calefacción y ascensores se reintegrarán el lunes al trabajo", ABC, 18 de julio de 1936.

La Vanguardia, 6 de agosto de 1936 (Suplemento).

\section{Otras fuentes}

Congreso de los Diputados, Diario de las Sesiones de Cortes, 58, 8 de julio de 1936. Consultado el 5 de febrero de 2019, https://app.congreso.es/est_sesiones/.

Instituto de Estadística, Estadísticas Históricas. Censos y padrones. Población de la Comunidad de Madrid por municipios. Consultado el día 5 de febrero de 2019, http://www.madrid.org/iestadis/fijas/estructu/general/otros/iserieshistoricas_ 
pob.htm

Sección de Estadística del Ayuntamiento de Madrid, Madrid: plano de sus vías. Madrid: Imprenta de las Artes Gráficas Municipales, 1934. Consultado el 5 de febrero de 2019, http://www.memoriademadrid.es/buscador.php?accion=VerFicha\&i$\mathrm{d}=128167 \&$ num_id=6\&num_total $=20$.

Ministerio de Trabajo, Sanidad y Previsión, "Orden disponiendo que los obreros de la construcción se reintegren al trabajo en todas las obras, tajos, fábricas y talleres, en que estuvieren ocupados, el próximo lunes 3", Gaceta de Madrid, 2 de agosto de 1936, 1002. Consultado el 5 de febrero de 2019, https:/www.boe.es/datos/pdfs/BOE//1936/215/B01002-01002.pdf.

Sesión de la Diputación Permanente de Cortes, sesión del miércoles 15 de julio de 1936, 6-7. Consultado el 5 de febrero de 2019, https://app.congreso.es/est_sesiones/.

\section{Fuentes secundarias}

González Calleja, Eduardo y Sánchez Pérez, Francisco. "Revisando el revisionismo. A propósito del libro 1936. Fraude y violencia en las elecciones del Frente Popular", Historia Contemporánea 58 (2018): 851-881.

Juliá Díaz, Santos. “¿Feudo de la UGT o capital confederal? La última huelga de la construcción en el Madrid de la República", Historia Contemporánea 6 (1991): 207-222.

. "Madrid, capital del Estado (1833-1933)". En Madrid. Historia de un capital, editado por Santos Juliá Díaz, David R. Ringrose y Cristina Segura Graíño, 315-576. Madrid: Alianza Editorial, 2006.

Montero Díaz, Julio y Cervera Gil, Javier. "Madrid en los años treinta. Ambiente social, político, cultural y religioso", Studia et Documenta: rivista dell'Istituto Storico San Josemaría Escrivá 3 (2009): 13-39.

Rodríguez Martín, Nuria. "La capital de un sueño: Madrid 1900-1936: la formación de una metrópoli europea". Tesis doctoral, Universidad Complutense de Madrid, 2013. 
Sánchez Pérez, Francisco. "La huelga de la construcción en Madrid (junio-julio, 1936)", Historia 16154 (1989): 21-26.

. "Clase obrera y conflictividad social en el Madrid del Frente Popular (febrero-julio de 1936)", Cuadernos de Historia Contemporánea 13 (1991): 47-71.

. "Madrid, capital de la protesta: de agosto de 1917 a julio de 1936", Cuadernos de Historia Contemporánea $N^{0}$ Extra 1 (2007), 301-311.

. "Madrid de preguerra, Madrid en huelga. ¿Vísperas de una revolución?". En 1936-1939, la Guerra Civil española: congreso internacional (Madrid 27, 28 y 29 de noviembre de 2006), sin paginar. Madrid: Sociedad Estatal de Conmemoraciones Culturales, 2008.

. "Las protestas del trabajo en la primavera de 1936", Mélanges de la Casa de Velázquez 41-1 (2011): 77-101

. "Movilización sociolaboral y oportunidades políticas en España y Francia durante la primavera de 1936, Nuestra Historia: revista e Historia de la FIM 1 (2016): 24-47.

Souto Kustrín, Sandra. «Y ¿Madrid? ¿Qué hace Madrid»: movimiento revolucionario y acción colectiva (1933-1936). Madrid: Siglo Veintiuno, 2004.

Tuñón de Lara, Manuel. "Prensa obrera e historia contemporánea". En Prensa Obrera en Madrid 1855-1936, editado por Santiago Castillo y Luis E. Otero Carvajal, 23-31. Madrid: Comunidad de Madrid y Alfoz, 1987.

Vadillo Muñoz, Julián. "Entre el pacto y la revolución. El movimiento libertario en la primavera de 1936", Nuestra Historia: revista de Historia de la FIM 1 (2016): 48-64. 